\title{
Efecto de la contaminación minera sobre el perifiton del río Guadiamar
}

\author{
Gonzalo Martín*, Eva Alcalá*, Carolina Solá**, Ángel Plazuelo*, Ma Dolores Burgos* y \\ Elisabeth Reyes* y Julia Toja*
}

(*) Dept. Biología Vegetal y Ecología. Universidad de Sevilla. Apartado 1095, 41080 SEVILLA (jtoja@us.es)

(**) Dept. Ecología. Universidad de Barcelona. Avd. Diagonal 645, 08028 BARCELONA (csola@porthos.bio.ub.es)

\section{RESUMEN}

Este trabajo presenta el efecto que la contaminación por metales pesados ha tenido sobre el perifiton del río Guadiamar y está englobado dentro de un seguimiento general de los organismos y la calidad del agua que se ha estado llevando a cabo desde el accidente de las minas de Aznalcóllar, en abril de 1998. Se pretende discernir qué factores tienen más importancia en la estructuración de la comunidad, hasta qué punto la concentración de metales ha llegado a alterarla y si hay bioacumulación de metales pesados en perifiton. Para ello se han contrastado las observaciones de campo con experimentación en el laboratorio, sometiendo en este caso al perifiton a distintas concentraciones de una mezcla de metales pesados (similares a las que ha sufrido el río), como único factor de alteración. Los resultados indican que las algas bioacumulan metales, que estos afectan al desarrollo y la composición de la comunidad, pero que en el río esta respuesta refleja más una adaptación a tolerar la acidez del agua que a la contaminación por metales pesados (posiblemente porque las especies más sensibles a los metales no se pueden desarrollar). Por tanto, la comunidad resultante en el río es diferente de la que se desarrolló en el laboratorio, ya que en este último sólo se seleccionaron especies en virtud de su mayor o menor tolerancia a la contaminación por metales pesados.

Palabras clave: Perifiton. Acidez. Metales pesados. Ríos.

\begin{abstract}
This paper shows the effects that pollution by heavy metals has had on the periphyton of the Guadiamar River and is included into a general follow-up of the biota and water quality, which has been taking place since the Aznalcóllar mine tailings spill, in April 1998. The objective is to know which factors have the most importance with regard to community structure, to which point the concentration of metals has come to alter it, and to know whether bioaccumulation of heavy metals occurs in periphyton, whether it has altered the taxonomic composition of the periphyton community. To achieve this, analyses of periphyton samples taken at the river have been compared with those gotten in laboratory experiments, in which algae from non-polluted parts of the river were subjected to different concentrations of heavy metals(similar to those that affected the river), as the only agent able to alter the community. Results indicate that the algae do bioaccumulate heavy metals, and that they affect the community development and composition. However, in the river this response reflects more an adaptation to acidity tolerance, rather than heavy metal pollution (possibly duo to the fact that heavy metal sensitive species can not develop). Therefore, the periphyton community existing at the river differs from the one gotten in the laboratory, because in this case the taxa were selected only in relation to their tolerance to heavy metals.
\end{abstract}

Keywords: Periphyton. Acidity. Heavy metals. Rivers.

\section{INTRODUCCIÓN}

El río Guadiamar es el último afluente por la margen derecha del río Guadalquivir. Es uno de los pocos ríos andaluces no regulados en su cauce principal. Está situado en la franja pirítica de la Sierra Morena y en su cuenca ha habido explotaciones mineras durante muchos siglos. La última explotación en activo, las minas de Aznalcóllar ha determinado durante muchos años una constante contaminación metálica del tramo medio-alto del río, aunque sus efectos no 


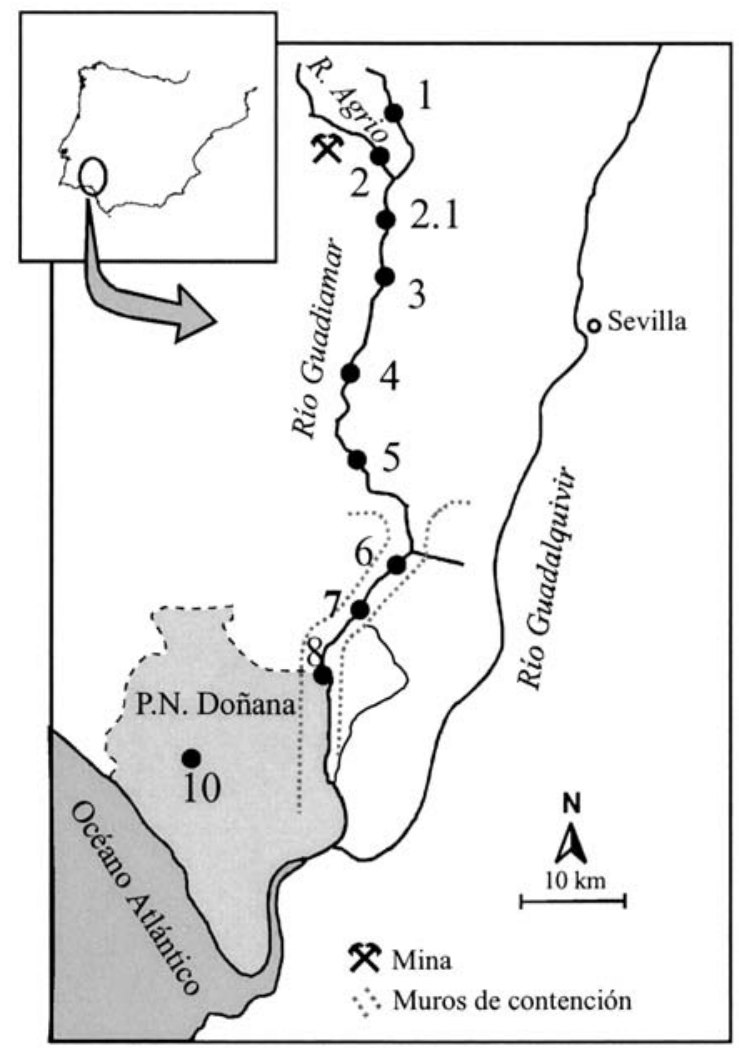

Figura 1. Localización del río Guadiamar y de las estaciones de muestreo. Los números de las estaciones que se han considerado en este trabajo se han destacado con mayor tamaño. Location of the Guadiamar River and the sampling stations. The number of stations considered in this paper are shown with a bigger size.

eran excesivamente notorios debido a la adaptación de las comunidades. Además de este impacto, el río Guadiamar recibía sin depurar las aguas residuales de los municipios ribereños, así como los vertidos de las industrias agropecuarias (alpechines y vinazas), que se concentran a partir del puente de las Doblas (Fig. 1, punto 3). Además, el tramo inferior del río, que se derramaba en la marisma de Doñana se modificó en los años 50, canalizándolo mediante la construcción de dos diques separados por $1 \mathrm{~km}$. (Arambarri et al., 1984).

Esta situación se agravó como consecuencia del accidente minero ocurrido en abril de 1998 cuando, por rotura de la balsa de estériles, se vertieron en el río (a través de su afluente el Agrio) $4 \mathrm{hm}^{3}$ de fango y aguas ácidas cargados de metales pesados, que asolaron todo el cauce y la zona inundable en una longitud de unos 40 Km. (Grimalt et al., 1999) A pesar de las labores de limpieza desarrolladas, tanto en los sistemas terrestres adyacentes, como en el propio cauce, aun ha quedado un resto de piritas que significan una contaminación a largo plazo (Gallart, et al., 1999).

El vertido tóxico, las labores de limpieza y otras actuaciones realizadas, tanto en el propio cauce como en los sistemas terrestres adyacentes, modificaron sensiblemente las características fisicoquímicas del agua y de los sedimentos. Estas variaciones afectaron, lógicamente, a todas las comunidades asociadas al río. Desde el punto de vista de las comunidades acuáticas, la situación de dichas poblaciones después de una pequeña recuperación inicial se mantuvo estática durante muchos meses, ya que tanto las condiciones físicas como las químicas no permitían una mejora sensible. Sin embargo, ya a mediados del año 2000 empezaron a producirse nuevos acontecimientos que han marcado un ligero cambio en el río Guadiamar. Por una parte, empezó la retirada de las trampas de sedimento, que habían convertido el río en una sucesión de pozas. Por otra parte se realizó la limpieza del cauce de la parte superior del río. Finalmente tuvieron lugar las primeras lluvias fuertes desde abril de 1998, con las consecuentes avenidas. El año 2001 fue mucho más lluvioso que los anteriores, especialmente en invierno, cosa que, en principio, fue favorable para la recuperación de las comunidades acuáticas.

Para controlar tanto el efecto del vertido como la posible recuperación se inició en Julio de 1998, apenas tres meses después de producido el accidente, un programa de seguimiento que ha producido ya algunas publicaciones (Prat et al., 1999; 2001; Solá et al., 2001)

En este artículo se reflejarán los cambios ocurridos en la densidad y composición de las comunidades de perifiton, así como en la acumulación en esta comunidad de elementos tóxi- 
$\cos (\mathrm{Zn}, \mathrm{Cu}, \mathrm{Pb}, \mathrm{Cd}, \mathrm{Tl}$, As y $\mathrm{Sb})$. Se utiliza el término perifiton en su acepción de algas bentónicas que se desarrollan adheridas a un sustrato (Wetzel, 1983, Iserentant, 1987, Casco, 1989).

En el campo coexisten diversos factores $(\mathrm{pH}$, concentración de metales, velocidad de corriente) que afectan a la estructura y composición taxonómica de las comunidades acuáticas. Además, los análisis de metales realizados en las muestras tomadas en el río no permiten diferenciar qué parte de estos metales está incorporada a la biomasa y qué parte corresponde a partículas de pirita acompañantes. Por esta razón se ha experimentado en el laboratorio, con un doble objetivo: determinar si hay bioacumulación de metales por parte de las algas y ver el efecto que la concentración de metales por sí sola tiene en la composición del perifiton. Los resultados obtenidos con estas experiencias también se recogen en este artículo.

\section{MATERIAL Y MÉTODOS}

\section{Experiencias de campo}

Aunque para el estudio general se seleccionaron 10 puntos de muestreo (Fig. 1) de los que dos: el 1 (zona fluvial) y el 10 (zona marismeña) se han utilizado como referencia al no haber estado afectados por el vertido tóxico, en este estudio sólo se consideran los resultados obtenidos en el tramo superior (puntos 1 a 3 ), debido a que en el tramo inferior el impacto de la contaminación orgánica enmascara el efecto del vertido tóxico. La frecuencia de muestreo ha sido variable a lo largo del periodo de estudio, siendo cada 6 semanas en 1998 y 1999, cada 3 meses en 2000 y cada 6 meses en 2001 .

Para el estudio del perifiton se recolectaron sustratos naturales colonizados. En el tramo del río considerado en el presente trabajo los sustratos recogidos fueron piedras en todos los casos, siempre en zona de corriente, si bien en otras zonas del río esto no fue así debido a la granulometría del sustrato y las características del cauce.
En cada punto se recogieron muestras para distintos tipos de análisis: 1) Análisis de pigmentos fotosintéticos como estima de la biomasa; 2) Identificación y recuento de especies; 3) Análisis de metales. Para cada uno de estos análisis se tomaron varias piedras al azar con una superficie total media de aproximadamente $100 \mathrm{~cm}^{2}$.

Para el análisis de pigmentos fotosintéticos los sustratos se introdujeron, directamente en el campo, en metanol. La extracción se realizó en oscuridad y a $4{ }^{\circ} \mathrm{C}$ durante 24 horas. La concentración de clorofila a se estimó por la fórmula de Talling \& Driver (Vollenweider, 1969).

Para la identificación y recuento de las especies de perifiton, los sustratos se introdujeron en formol al $4 \%$, en el laboratorio el sustrato se rascó y el material recogido se guardó en formol al 4\%, separándolo en dos alícuotas, una para la identificación de las especies y otra para el recuento utilizando la técnica Utermöhl (1958). A partir de estos recuentos se determinó la abundancia relativa de cada una de las especies.

Para el análisis de metales, los sustratos se introdujeron en agua MilliQ conservándose en frío. El raspado de los sustratos se realizó utilizando material previamente lavado con ácido nítrico para eliminar posibles restos de metales en él. Posteriormente, la suspensión en agua MiliQ obtenida se filtró en nitrato de celulosa WHATMAN de $0.45 \mathrm{~mm}$ de poro, congelando y liofilizando posteriormente los filtros. Las muestras biológicas liofilizadas se digirieron en recipientes cerrados (reactores de presión "Savillex", mod 561 2R) con $1.5 \mathrm{ml}$ de $\mathrm{HNO}_{3}$ y $0.4 \mathrm{ml} \mathrm{de} \mathrm{H}_{2} \mathrm{O}_{2}$ a $90^{\circ} \mathrm{C}$ durante 6 horas, diluyendo el resultado en $3 \mathrm{ml}$ de agua bidestilada. El mismo procedimiento se realizó al material de referencia adecuado. El análisis de metales se realizó por espectrometría de Masas de Plasma, acoplado inductivamente (espectrómetro IOP-MS Perkin-Elmer modelo Elam-6000), utilizando Rh como estándar interno. Todo el material utilizado en el tratamiento de las muestras para el análisis de metales fue previamente lavado con $\mathrm{HNO}_{3}$ al $10 \%$ y enjuagado varias veces con agua bidestilada. 
Los elementos que contaminan básicamente el Guadiamar y el Agrio, como efecto del accidente minero, son $\mathrm{Zn}, \mathrm{Cu}, \mathrm{Pb}, \mathrm{Cd}, \mathrm{Tl}$, As y $\mathrm{Sb}$, por lo que son los que se han analizado en las muestras. Entre ellos hay dos ( $\mathrm{Sb}$ y As) que son metaloides, pero, de forma general, se hablará de metales pesados.

Mediante un análisis canónico de correspondencias (CCA, PC-ORD), se ha comparado la abundancia de los taxa de perifiton dominantes en los distintos tramos de río, con diversas variables indicadoras de la contaminación minera ( $\mathrm{pH}$ y concentraciones de $\mathrm{Zn}, \mathrm{Pb}, \mathrm{Cd}, \mathrm{Cu}, \mathrm{Tl}$ ). No se han considerado los elementos no metáli$\cos$ (As y Sb), porque su comportamiento frente al $\mathrm{pH}$ es distinto a los elementos metálicos, de forma que las máximas concentraciones derivadas del accidente minero se han registrado en zonas situadas aguas abajo del tramo de río considerado en este trabajo (Toja et al., 2003).

\section{Experiencia de laboratorio}

En el experimento se ha procurado reproducir, dentro de lo posible, las condiciones naturales

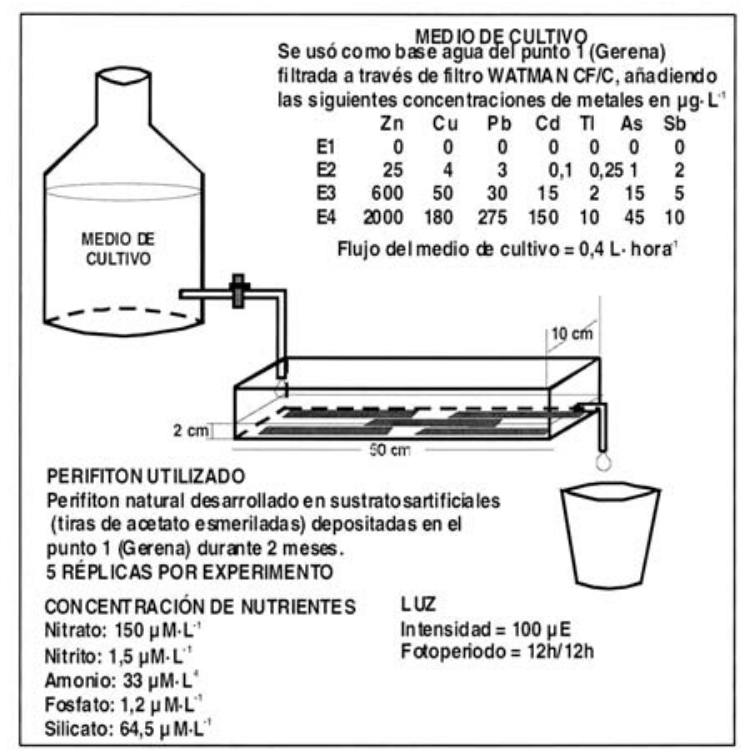

Figura 2. Diseño experimental en laboratorio. Laboratory experimental design. del río (Fig. 2). Para ello se han utilizado comunidades de perifiton naturales, desarrolladas en el punto de referencia (Gerena, punto 1) sobre sustratos artificiales, consistentes en tiras de acetato esmeriladas, que se mantuvieron en el río 4 semanas. Como base del medio de cultivo, se utilizó agua procedente de Gerena, previamente filtrada con filtros WATMAN GF/C. Se enriqueció el agua con nutrientes (compuestos de nitrógeno y fósforo) hasta alcanzar concentraciones similares a las máximas que de los mismos se han detectado en el río en Gerena a lo largo del estudio (Prat et al., 1999). La razón de este enriquecimiento era que, en ensayos previos, se había visto que, dado que el agua en Gerena tiene, normalmente, pocos nutrientes, las comunidades decaían por limitación de éstos antes que por el efecto tóxico. La experimentación se realizó en una serie de pequeños canales (50 $\mathrm{cm}$ de largo, 10 de ancho y una lámina de agua de $2 \mathrm{~cm}$.) haciendo pasar el agua por ellos en flujo continuo de 0.4 litros/hora, similar al que se encuentra en las pozas del río, donde se colocaron los sustratos artificiales. La temperatura se mantuvo entre 20 y $23{ }^{\circ} \mathrm{C}$, la intensidad de luz fue de $100 \mu \mathrm{E}$ y el fotoperiodo de 12 horas luz/12 horas oscuridad.

Se colocaron 5 tiras colonizadas en cada uno de los 4 canales utilizados. Uno de los canales se utilizó como control, sin adición de metales y a los demás se les añadió una mezcla de los metales considerados en este estudio ( $\mathrm{Zn}, \mathrm{Cu}$, $\mathrm{Pb}, \mathrm{Cd}, \mathrm{Tl}, \mathrm{As}, \mathrm{Sb})$ en concentraciones que, aproximadamente, eran similares a las mínimas (ensayo E1), media (ensayo E2) y máxima (ensayo E3), detectadas para cada metal en el agua del río. Las concentraciones utilizadas en cada ensayo se especifican en la figura 2.

El crecimiento del perifiton se determinó por la medida de la clorofila $a$, tomando una muestra de cada uno de los 5 sustratos al inicio de la experimentación (tiempo 0) y semanalmente durante cinco semanas. Para la identificación de las especies y el contenido en metales se tomaron muestras con la misma periodicidad. La metodología utilizada fue la seguida para las muestras de campo. 


\section{RESULTADOS}

\section{Crecimiento y composición de la comunidad perifítica}

\section{Estudio en el campo}

La figura 3 muestra la concentración de clorofila en el tramo considerado (puntos 1, 2, 2.1 y 3). Los resultados obtenidos muestran una dinámica marcadamente estacional y, en general, son algo erráticos. Además de las variaciones estacionales en las características medioambientales, la razón de esta irregularidad puede estar en el hecho de que los sustratos se tomaban al azar y, teniendo en cuenta que la distribución del perifiton es contagiosa (sobre todo en lo que se refiere a las algas filamentosas), puede que las muestras tomadas no reflejaran fielmente las características medias de la zona. Pero, en general, salvo en los primeros momentos tras el accidente o en diversos eventos ligados a las actuaciones en el cauce, en las zonas afectadas no sólo no se ha registrado una disminución del crecimiento, sino que en muchas ocasiones ha sido superior a la de la zona de control. La razón está en la composición de las comunidades. En los puntos afectados rápidamente se estableció una comunidad de algas adaptadas a soportar las nuevas condiciones, siendo la mayoría de macroalgas filamentosas, Ulothrix spp (aunque alguna de estas especies podría pertenecer al género Klebsormidium) y Mougeotia sp, principalmente, que incrementan mucho la concentración de clorofila.

La figura 4 muestra los niveles de metales registrados en las muestras de perifiton. La dinámica seguida ha sido bastante compleja. En general se observa un descenso a finales del periodo de estudio para casi todos los elementos tóxicos analizados. En parte puede deberse al descenso en la concentración de metales en el agua pero también puede deberse a que los dos últimos años fueron lluviosos, con avenidas que aumentaron la velocidad del agua y, como consecuencia, lavaron parte de la materia inorgánica depositada en los biofilms. En cualquier caso, es patente el hecho de que las concentraciones de

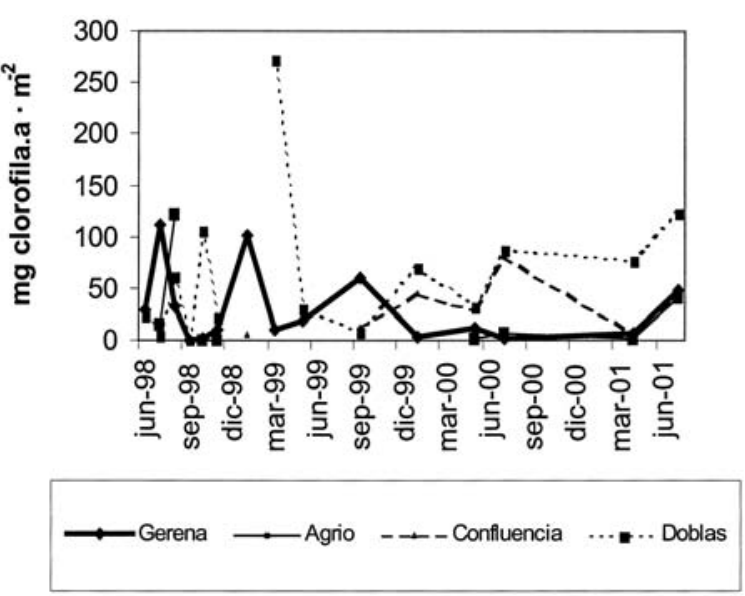

Figura 3. Concentraciones de clorofila a en el perifiton, a lo largo del tiempo, en cada una de las estaciones de muestreo estudiadas. Periphyton chlorophyll a concentrations along the study period, in each sampling station.

metales de las zonas afectadas han sido y todavía son muy superiores a la zona control.

La figura 5 muestra la composición de la comunidad de perifiton en el tramo considerado a lo largo del tiempo, diferenciando los periodos de avenida y sequía ya que, por causas naturales, son bastante distintos. El punto control (Gerena) está caracterizado durante la inundación por diatomeas: Achnanthes minutissima y Gomphoneis olivacea principalmente pero, también en mayor o menor medida según el año, Cyclotella meneghiniana, Melosira varians, algunas Synedra (S. ulna, S. acus), Cocconeis placentula, y varias especies de los géneros Navicula (N. cryptocephala, N. decussis), Cymbella (C. amphicephala, C. ventricosa, $C$. caespitosa, C. helvetica, C. affinis), Gomphonema ( $G$. angustatum, $G$. constrictum) y Nitzschia (N. palea, N. sinuata, N. hungarica). En sequía dominan Cianofitas representadas mayoritariamente por Oscillatoria spp, Calothrix sp y Geitleribactron periphyticum. El punto 2 (río Agrio) es el que más sufre la acción de la mina. Las especies más características de la zona son Klebsormidium sp, Ulothrix sp, Mougeotia sp y varias diatomeas: Achnanthes minutissima, Eunotia exigua, Nitzschia palea y Naviculáceas (Pinnularia biceps y Navicula spp 


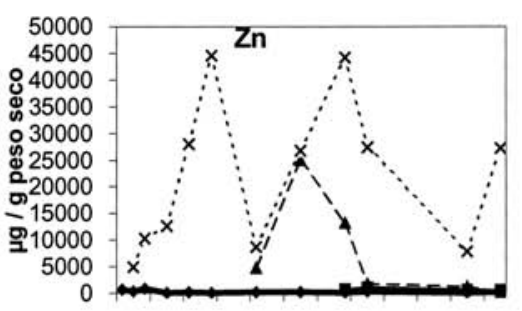

.

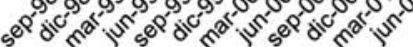

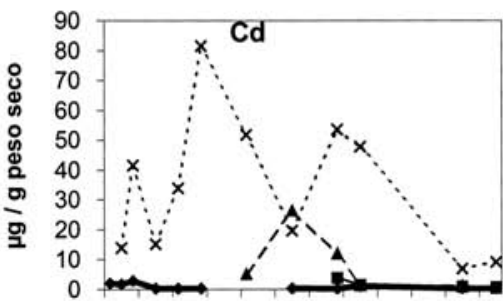

,

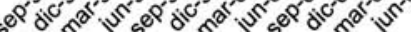

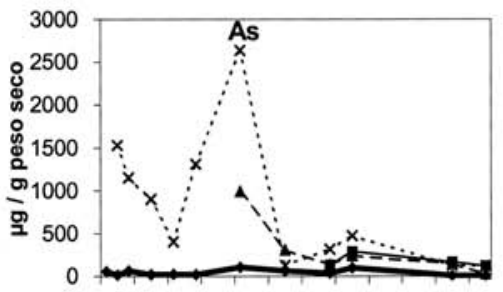

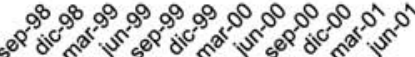

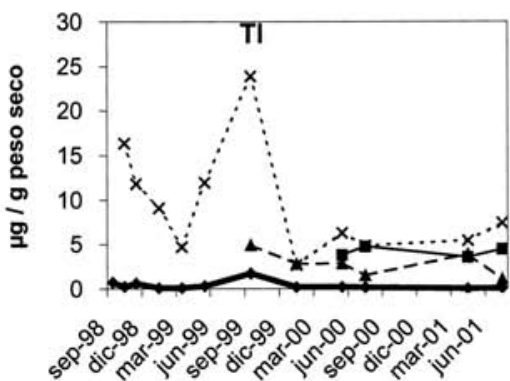

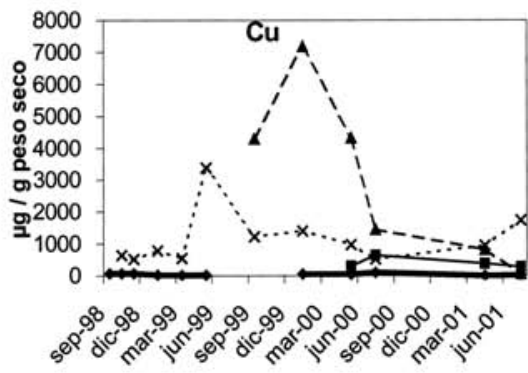

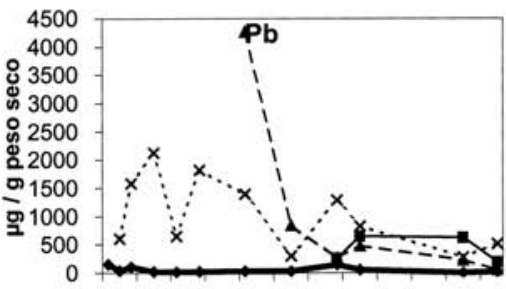

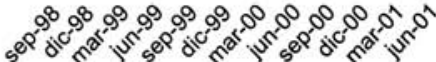

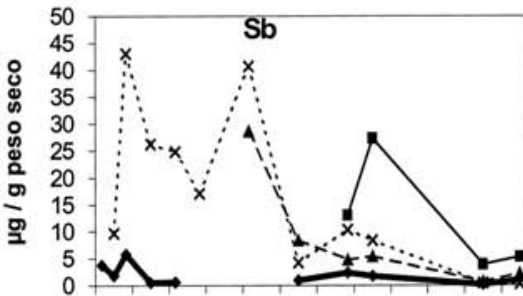

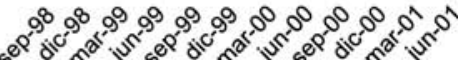

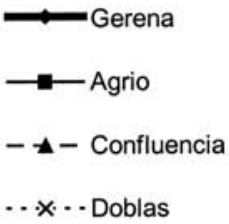

Figura 4. Contenido de cada uno de los metales pesados en el perifiton, a lo largo del tiempo, en cada una de las estaciones de muestreo estudiadas. Heavy metal contents in the periphyton along the studied period, in each sampling station

sobre todo). En función del año y la estación las proporciones de estos taxa han variado mucho aunque, como norma general, las filamentosas han significado gran parte de la biomasa. El punto 2.1 (confluencia) presenta en su mayor parte diatomeas. Se han observado desarrollos 

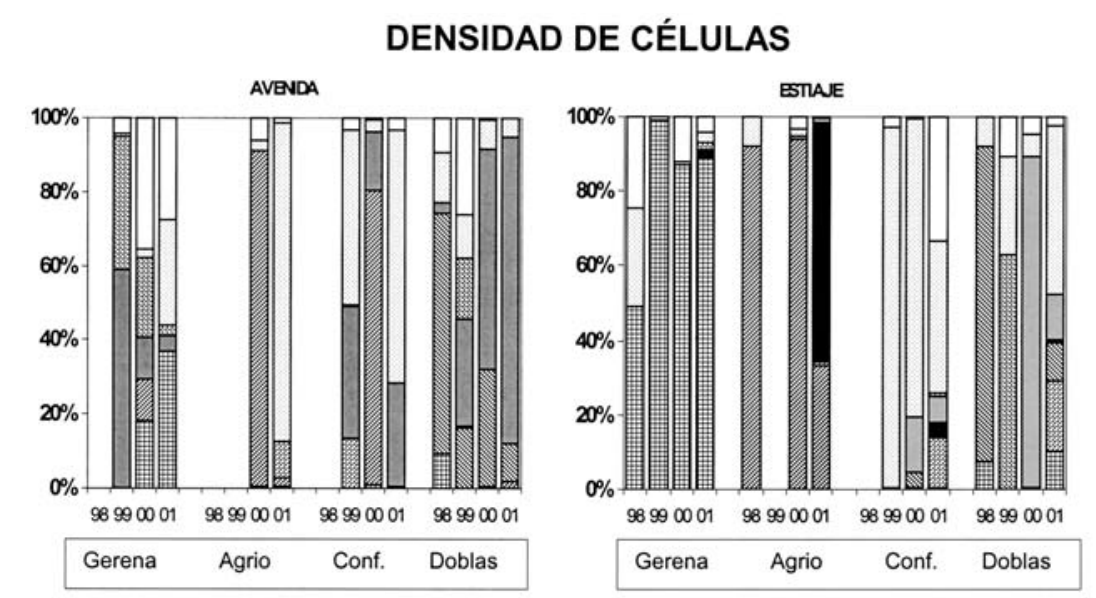

\section{BIOVOLUMEN}
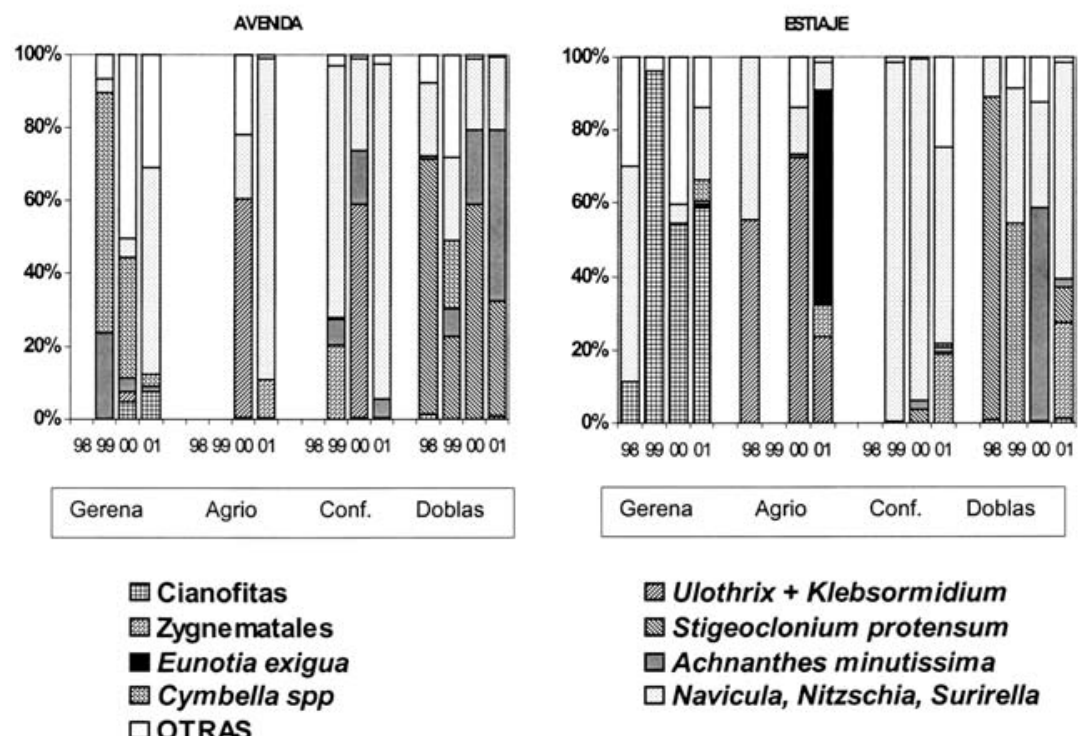

Figura 5. Abundancia relativa media, en cada periodo considerado (avenida a la izquierda, estiaje a la derecha) entre los años 1998 y 2001, de las especies o grupos de especies dominantes en cada una de las estaciones de muestreo. Se representa la abundancia del número de individuos (arriba) y del biovolumen (abajo). Relative average abundance of the dominant species or group of species found in each considered period (wet season on the left and dry season on the right) from 1998 to 2001. The abundance of number of individuals (up) and biovolume (down) are shown.

masivos de filamentosas, Ulothrix, Klebsormidium y Mougeotia en buena parte de los casos, principalmente en estiaje. Estos desarrollos han aportado mucha biomasa y clorofila al sistema, aunque la observación al microscopio revelaba mayor efectivo numérico poblacional de otras especies (principalmente Achnanthes minutissima, Eunotia exigua, Navicula sp, Pinnularia biceps, Nitzschia palea y Surirella ovata). En el punto 3 (Las Doblas) Stigeoclonium protensum sustituye a las filamentosas encontradas aguas arriba. Esta especie, que fue la primera que colonizó esta zona del río tras el accidente (Prats et al., 1999), es la que más biomasa llega a aportar y aparece acompañada por diversas diatomeas (Achnanthes minutissima, Surirella ovata, Nitzschia palea y, en menor medida, Navicula spp y Gomphonema angustatum. 


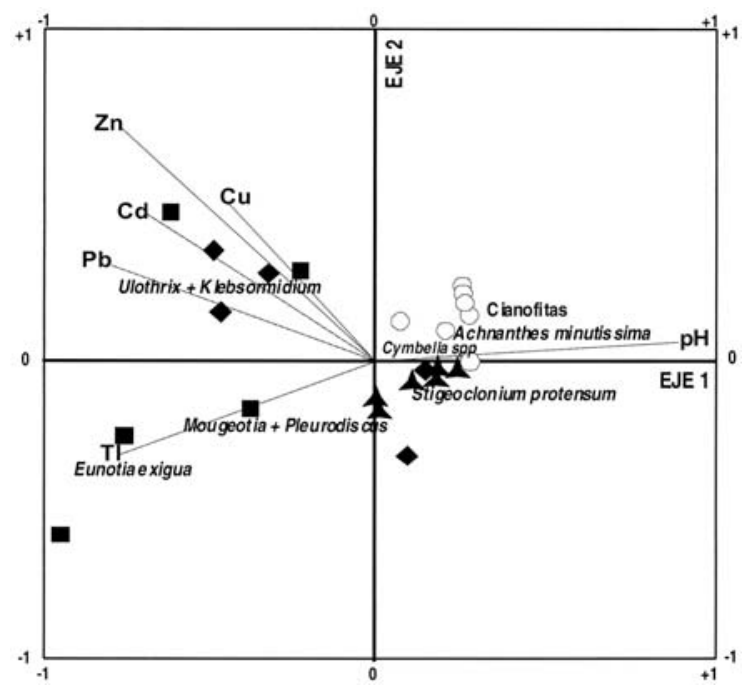

Figura 6. Distribución en el plano definido por los dos primeros ejes del análisis CCA, de las muestras de perifiton, de las variables fisicoquímicas y de los taxa considerados. Las muestras correspondientes a cada estación se han indicado con símbolos: (○) Punto 1, Gerena; (ם) Punto 2, Agrio; $(\bullet)$ Punto 2.1, Confluencia; (ᄉ) Punto 3, Doblas. Distribution, of the samples, the physical and chemical variables, and the considered taxa, in the plane defined by the two first CCA axes. Samples corresponding to each sampling station are indicated by symbols: (O) Station 1, Gerena; (ם) Station 2, Agrio;

Station 2.1, Confluencia; (ᄉ) Station 3, Doblas.

La figura 6 muestra la distribución de los taxa dominantes de perifiton y de las muestras consideradas en el plano definido por los dos primeros ejes del análisis CCA. El primer eje, que adsorbe el $22.5 \%$ de la varianza, está definido por el $\mathrm{pH}$. El segundo eje, que adsorbe el $15.9 \%$ de la varianza, está definido por la oposición entre el talio y el resto de metales.

El eje 1 separa claramente las muestras correspondientes al punto 1 (Gerena) y a la mayoría de las del punto 3 (Las Doblas) de todas las del punto 2 (Agrio) y de la mayoría de las del punto 2.1 (Confluencia). Pero algunas de las muestras de la Confluencia se confunden con las de las Doblas. Esto está ligado normalmente a que el caudal en las Doblas proceda mayoritariamente del Guadiamar o del Agrio. En el primer caso, la calidad del agua aumenta en la Confluencia y en el segundo disminuye en las Doblas. Según este
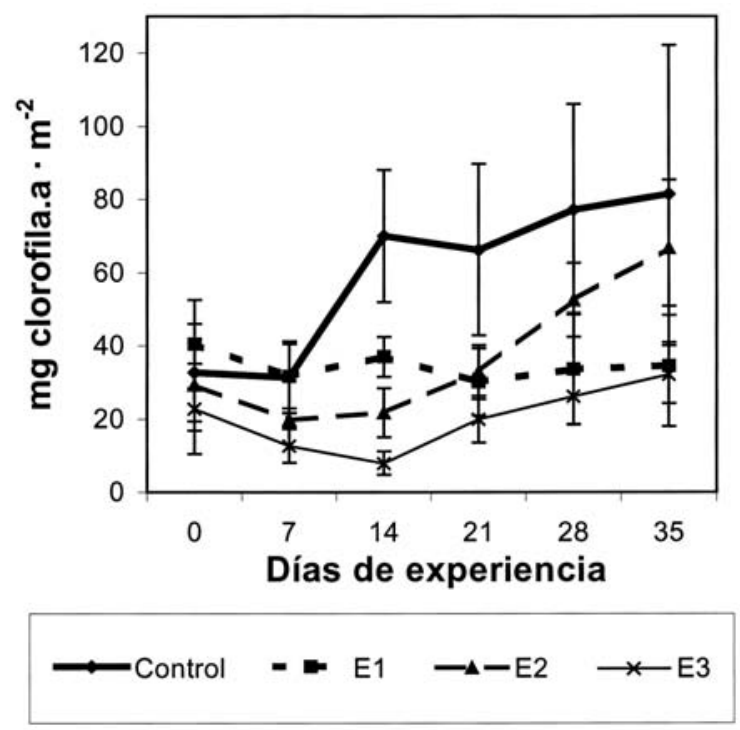

Figura 7. Crecimiento de la comunidad (estimado por la concentración de clorofila.a) en los distintos ensayos (E1, concentración de metales baja; E2, intemedia; E3, alta). Se muestra la desviación estándar en cada caso. Community growth (estimated by the chlorophyll a concentration) in the different assays (E1, low concentration of heavy metals; E2, intermediate; E3, high). Standard deviation is shown in each case

primer eje, los taxa se diferencian en dos grupos: por un lado el grupo de las Cianofitas (Calothrix $\mathrm{sp}+$ Lyngbya $\mathrm{sp}+$ Oscillatoria spp), Achnanthes minutissima, Cymbella spp y Stigeoclonium protensum y, por otro a las Ulotricales (Ulothix + Klebsormidium), Zignematales (Mougeotia + Pleurodiscus) y Eunotia exigua que son especies tolerantes a la acidez.

Aunque las muestras de Gerena y las Doblas aparecen relativamente juntas, el segundo eje determina una ligera separación, caracterizando Gerena por las Cianofitas, Achnanthes munutissima y Cymbella spp y a las Doblas por Stigeoclonium protensun. Como se ha comentado anteriormente, esta especie fue la primera que colonizó el tramo de las Doblas en los primeros meses posteriores al accidente minero, en cuanto se neutralizó el pH del agua. Por lo tanto, parece que es una especie que tolera una cierta cantidad de metales, pero no resiste la acidez. Por otro lado, las Cianofitas prácticamente sólo 
aparecen en Gerena. Achnanthes minutissima y Cymbella spp, aunque sean proporcionalmente más importantes en Gerena son abundantes en ambas zonas del río. En el caso de los puntos más contaminados, el segundo eje diferencia claramente al grupo de las Ulotricales de Eunotia exigua y, en menor medida, de las Zignematales. Es posible que estas especies sean tolerantes a concentraciones relativamente altas de talio, mientras que para las Ulotricales sea muy tóxico, pero no hemos encontrado referencias bibliográficas al respecto.

\section{Experiencias en el laboratorio}

El crecimiento del perifiton se ha visto claramente influenciado por la adición de la mezcla de metales pesados (Fig. 7). El control ha registrado siempre un crecimiento mayor que los otros ensayos, salvo en la primera semana, que fue un periodo de aclimatación de las algas al ambiente del laboratorio y de respuesta a las condiciones impuestas. Aunque con oscilaciones, el crecimiento en el control se estanca hacia las dos semanas de experimentación, posiblemente por limitaciones de espacio. En todos los demás ensayos, el desarrollo ha sido tanto menor cuanto mayor ha sido la concentración de metales. También en estos casos se observa un cambio a partir de los 14 días de experiencia, pero es diferente según el ensayo. A partir de este momento, E1 se mantiene más o menos estable (aunque en este caso no puede achacarse a limitación de espacio) pero, tanto en E2 como en E3, empieza un crecimiento que dura hasta el final de la experimentación, aunque no se llegue nunca a los niveles del control.

La figura 8 ilustra la evolución temporal de la concentración de metales en el perifiton para los distintos ensayos a lo largo del tiempo. Para todos ellos, salvo para el As, se observa una pequeña bajada a lo largo del tiempo en el control. Ello se debe al lavado, por el flujo de agua filtrada, de las partículas inorgánicas depositadas sobre el perifiton. Hay que tener en cuenta que, aunque no afectado por el accidente minero, el punto 1 , en el que se dejaron colonizar los sustratos artificiales para experimentación, recibe aportes de la cuenca pirítica, donde también hay algunas minas abandonadas, por lo que los sedimentos que recibe también tienen una cierta cantidad de metales. De forma general se observa que la concentración de metales en la biomasa es directamente proporcional a la adición de metales al agua y que tiende a aumentar en el tiempo. As muestra una dinámica distinta porque los valores que se registran en el agua del punto de referencia, la usada en el sistema de experimentación, no son significativamente distintos de los de la zona contaminada, ya que este elemento no ha ido tan ligado a la contaminación minera como el resto.

Si se comparan los niveles de metales alcanzados en el experimento con los registrados en el campo, se observa que en éste último son un orden de magnitud superiores, lo que confirma que una parte de los metales que hay en los biofilms del río corresponde a materia inorgánica (restos de piritas). Pero las diferencias observadas en el experimento entre el control y los demás ensayos indican que sí hay una bioacumulación.

La comparación de los distintos tratamientos muestra que la composición taxonómica de la comunidad ha variado sensiblemente (Fig.9) por los metales añadidos. Las cuatro fotografías de la figura 10 muestran la fisonomía del perifiton al final del experimento para los distintos ensayos. Sin embargo, el patrón de variación no ha sido el mismo que se ha observado en el campo, donde hay otros factores como, por ejemplo, la velocidad del agua, el pH y la depredación que pueden tener tanta o más incidencia que los metales en la estructuración de la comunidad.

El control es rico en diatomeas durante todo el periodo de experimentación. Las especies dominantes y más características de este grupo son Achnanthes minutissima y varias Cymbella (C. caespitosa, C. helvetica, $C$. amphicephala, C. affinis). Aunque A. minutissima supere con creces en efectivo numérico a Cymbella spp, el aporte en biomasa de estas últimas es importante. La adición de metales determina distintos efectos patentes, sobre todo, al término de la experimentación. 

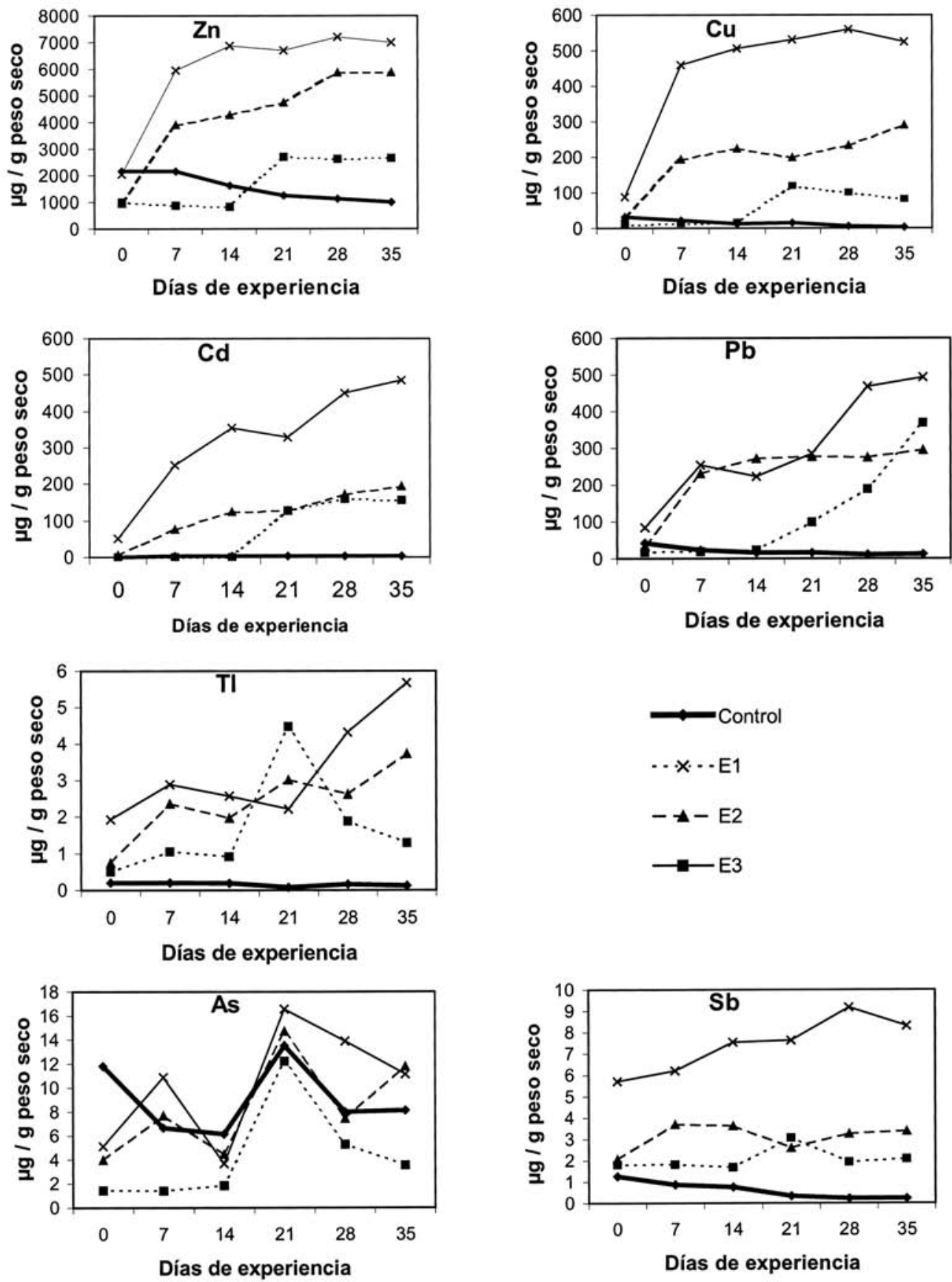

Figura 8. Evolución de la concentración de metales ( $\mu \mathrm{g} \cdot \mathrm{g}^{-1}$ de peso seco), en el perifiton de los distintos ensayos. La desviación estándar de las muestras se ha mantenido en la práctica totalidad de los casos menor del 30\%. Evolution of the concentration of heavy metals $\left(\mu \mathrm{g} \cdot \mathrm{g}^{-1} \mathrm{DW}\right)$ in periphyton in the different assays. Standard deviation values were less than $30 \%$ in most cases. 


\section{DENSIDAD DE CÉLULAS}

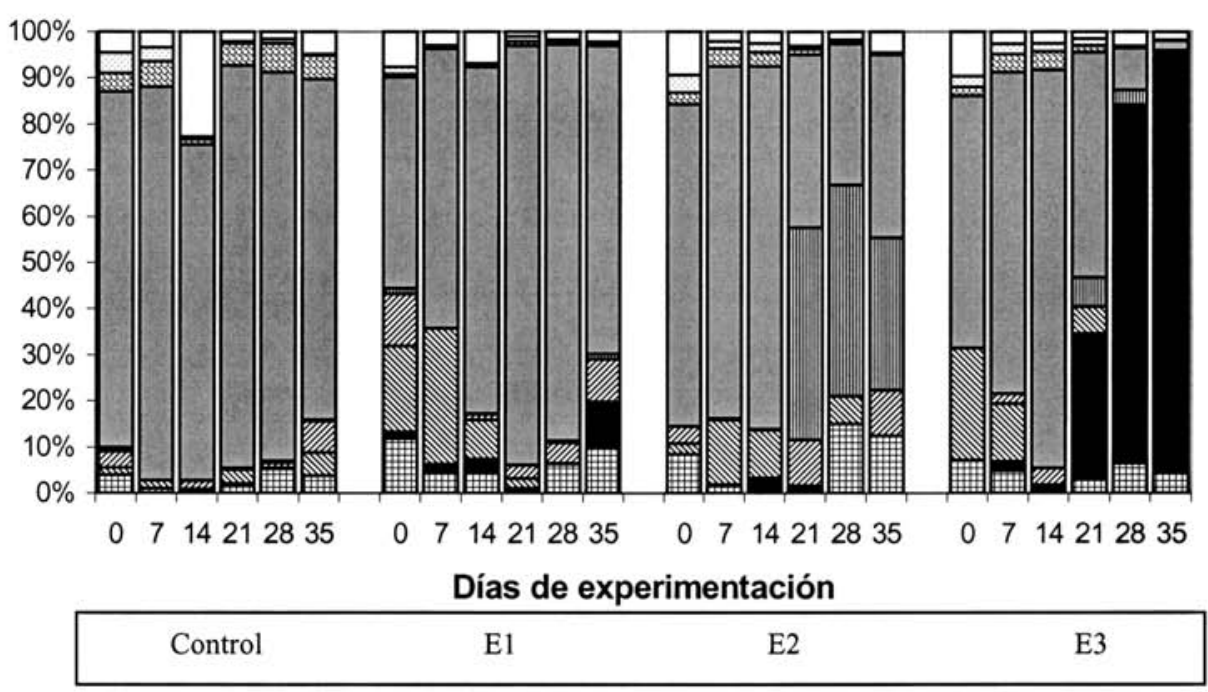

BIOVOLUMEN

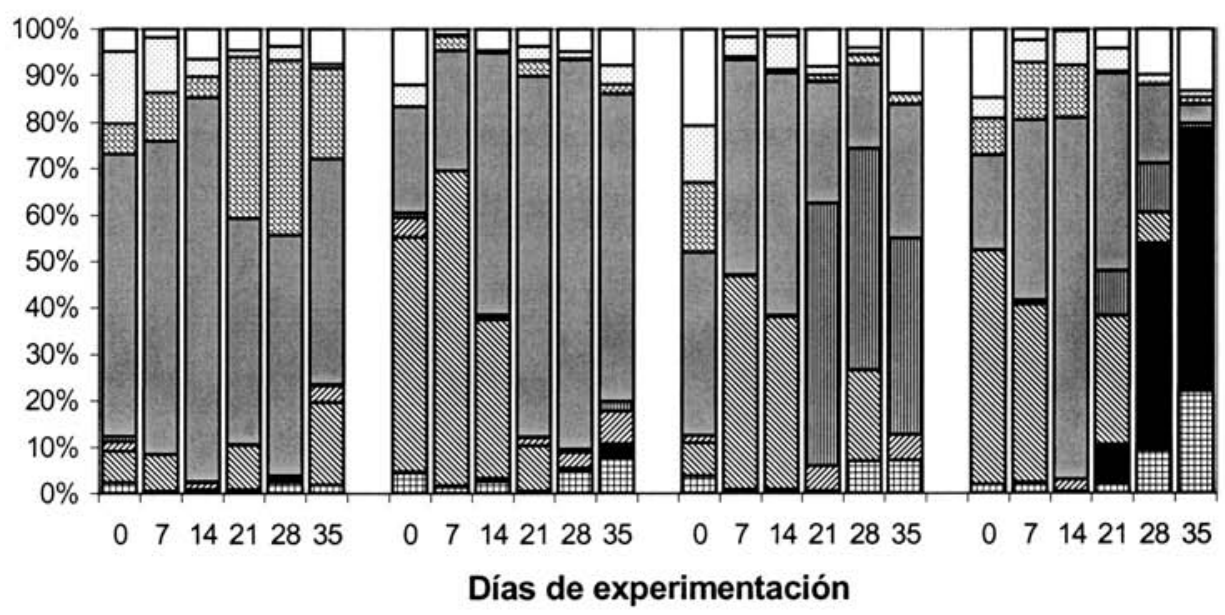

\begin{tabular}{|llll|}
\hline Control & E1 & E2 & E3 \\
\hline
\end{tabular}

\begin{tabular}{|c|c|c|}
\hline ⿴囗十 Lyngbya sp & Microcystis sp & 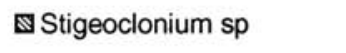 \\
\hline VUlothrix sp & 口Clorococal & 口Achnanthes minutissima \\
\hline M Cymbellaceae & 口Otras diatomeas & 口OTRAS \\
\hline
\end{tabular}

Figura 9. Proporciones relativas, en densidad de individuos (arriba) y en biovolumen (abajo), entre los taxa dominantes en el perifiton en el laboratorio a lo largo del tiempo para los distintos ensayos. Relative proportions of individuals' densities (up) and biovolume (bottom) among the dominant taxa in the periphyton at the laboratory over time for the different assays. 
De forma general, se observa una disminución en la riqueza de diatomeas, especialmente en las Cymbella. A. minutissima es la diatomea más tolerante a las condiciones impuestas. Los distintos tratamientos presentan peculiaridades interesantes: E1 pasa a ser dominado, tanto en biomasa como en densidad, por $A$. minutissima . En E2 aparecen al final de la experimentación pequeñas clorococales (Monoraphidium, Scenedesmus, especies afines a Chlorella) y algunas cianofitas (Lyngbya sp). Por su parte, E3 pasa a ser dominado claramente por Lyngbya y Microcystis que desarrollan vainas mucilaginosas. Aunque en términos de efectivo numérico Microcystis parece mucho más abundante que Lyngbya, ello obedece a que el recuento se ha hecho por células para Microcystis y por filamentos para Lyngbya; la diferencia se reduce si se comparan biovolúmenes.

\section{DISCUSIÓN}

Los factores que más han discriminado la composición de la comunidad en el campo y en el laboratorio no han sido los mismos. En el laboratorio las algas únicamente responden a la concentración de metales, pues éste es el factor que ha diferenciado los tratamientos. Las más sensibles (Cymbella) desaparecen con el paso del tiempo en E1, E2 y E3, quedando sólo las más resistentes (Achnanthes minutissima, por ejemplo). Esta especie, dominante en E1, es sumamente adaptable y tolerante. Generalmente es la dominante en muchos ecosistemas acuáticos de la Sierra Norte (Casco, 1989; Toja y Casco, 1991, Casco y Toja, 2003) donde el nivel natural de metales es relativamente alto, debido a la franja pirítica.

La estrategia observada en el laboratorio, cuando las concentraciones de metales son altas, es la de seleccionar especies como las de los géneros Microcystis y Lyngbya, capaces de aislarse del medio a través de una capa de mucílago que minimice la transferencia de metales hacia las algas (Admiraal et al., 1999). Aunque no es habitual la presencia de Microcystis en el fitobentos, la razón de su aparición en los ensayos de laboratorio puede radicar en que el flujo de agua durante la experimentación fue lo suficientemente débil como para permitir su crecimiento y en que el mucílago posibilitaba la proliferación en un medio con metales pesados. El crecimiento de Lyngbya y Microcystis es el res-
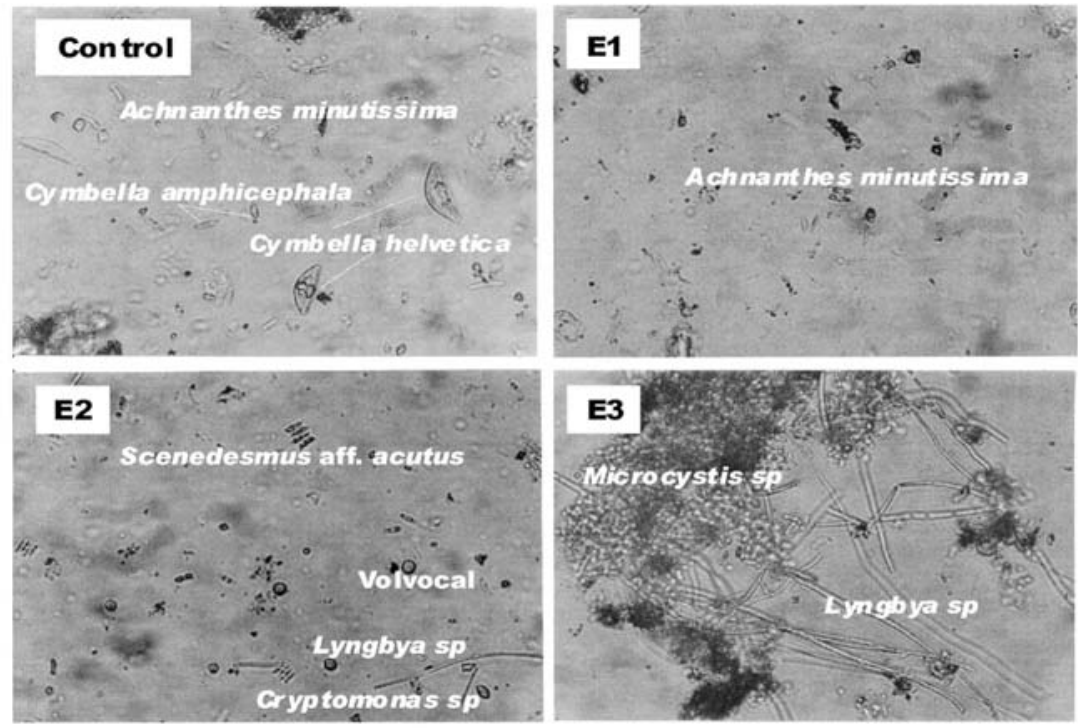

Figura 10. Fisonomía del perifiton al final del experimento en los distintos ensayos. Appearance of periphyton at the end of the experiment in the different assays. 
ponsable del aumento de clorofila que se registró en E2 y E3 a partir de los 14 días. Si bien el biofilm llega a bioacumular metales, ya que la concentración de éstos en biomasa es proporcional a la concentración en agua (Fig. 5) y aumenta en el tiempo, muy posiblemente una parte importante de ellos queda retenida en la estructura mucilaginosa.

En el Agrio, durante el primer año y medio posterior al accidente, el desarrollo de las comunidades fue errático, debido no sólo a la contaminación sino a las modificaciones del cauce. A partir del 2000 se ha implantado una comunidad de algas bien adaptadas a los efectos de la mina.

Haciendo un paralelismo, teniendo en cuenta la concentración de metales, entre los distintos ensayos de laboratorio y puntos de muestreo en el campo, el Agrio corresponde al ensayo E3. Sin embargo, la comunidad es totalmente diferente. En el Agrio no hay Cianofitas, posiblemente porque son incapaces de tolerar el $\mathrm{pH}$ ácido. Además, se conoce de ellas que son sensibles a los metales pesados por lo que, para subsistir, están obligadas a aislarse del medio con una capa mucilaginosa, como se observa en el laboratorio. En el río esta estrategia no parece viable. Puede haber dos causas que destruyan la estructura mucilaginosa: la depredación y la velocidad de corriente. Se ha comprobado, además, que este último factor aumenta la toxicidad de los metales por varias causas (Navarro, 2001).

Este efecto de la corriente se debe, por un lado, a que la velocidad alta estimula la fotosíntesis (Pfeifer y McDiffet, 1975) y la incorporación de nutrientes (Whitford y Schumacher, 1961) y parece que hay una relación entre la incorporación de metales y el incremento de la fotosíntesis (Navarro et al., 2000). Por otro lado un aumento de velocidad disminuye el grosor de la capa límite, lo que se traduce en una mayor accesibilidad de los nutrientes al perifiton (Stevenson y Glover, 1993) y, probablemente, de los metales. La absorción de metales se lleva a cabo mediante un mecanismo bifase (Genter, 1996). La primera es física (bioabsorción) y la segunda depende del metabolismo.
Por otro lado, la depredación sobre el perifiton puede alterar las cubiertas mucilaginosas, haciendo más asequibles los metales para su incorporación a la biomasa de algas, como se ha demostrado con otros tóxicos como la antrazina (Muñoz, com. personal).

Así, lejos de encontrar Cianofitas con mucílagos, en el campo se hallan especies muy tolerantes al $\mathrm{pH}$ ácido como varias del género Ulothrix y/o Klebsormidium, Mougeotia sp, Pleurodiscus sp y Eunotia exigua. Ulothrix y Klebsormidium son géneros típicos de sistemas sometidos a este tipo de tensión. Ciertos estudios han denominado como Ulothrix y otros como Hormidium o Klebsormidium a los largos filamentos de Ulotricales propios de este tipo de sistemas, cuando posiblemente se haya tratado en todos los casos del mismo género (Niyogi et $a l$, 2002). Las Zignematales, como Mougeotia, son frecuentes en lagos ácidos (Prescot, 1962; Planas, 1996) y, aunque en general existen taxa de diatomeas que resisten la acidez, el género Eunotia parece estar especialmente favorecido (Moss, 1973; Mulholland et al., 1986; Marker y Willoughby, 1988; Vinebrooke y Graham, 1997; Kapfer, 1998; Niyogi et al., 2002). Las Zignematales se desarrollan mejor con velocidades pequeñas de corriente (épocas de estiaje), mientras que las diatomeas están favorecidas por velocidades mayores (épocas de avenida) (Folch, 1985). Por lo tanto, el efecto puede ser debido más a la acidez del agua que a las concentraciones de metales, aunque, lógicamente, también deben ser tolerantes a concentraciones elevadas de metales. Además, el análisis CCA parece indicar que Eunotia exigua es bastante tolerante a concentraciones relativamente altas de talio, pero no ocurre lo mismo con las Ulotricales. Pero no hemos encontrado referencias que abonen esta hipótesis.

De todas formas, los resultados obtenidos en los experimentos sí apoyan la hipótesis de que las altas concentraciones de metales son tóxicas para muchas algas, ya que muchas de las especies que colonizaron los sustratos en Gerena, desaparecieron en los experimentos al ser tratadas con metales (Cymbella, Gomphonema y Gomphoneis de 
forma más evidente). En estos experimentos, el $\mathrm{pH}$ se mantuvo siempre por encima de 7. Además, los experimentos se han llevado a cabo con velocidades de corriente muy bajas, con lo que la toxicidad que ya tienen los metales por sí solos se acentuaría en sistemas con más corriente.

Existen numerosos trabajos sobre el efecto del $\mathrm{Cu}$ sobre la estructura y el metabolismo de la comunidad algas (Eichenberger et al., 1981; Leland y Carter, 1984, 1985; Pratt y Rosenberger, 1993; Navarro, 2001). Existen también algunos trabajos sobre el Cd. Pero el resto de metales ha sido poco ensayado y mucho menos el efecto combinado de varios metales. Por ahora nuestros datos permiten afirmar que ha habido cambio en las comunidades y bioacumulación de metales en biomasa, pero no el papel que cada uno de los metales ha jugado en ello o si ha habido efectos sinérgicos.

En conclusión, la contaminación tóxica ha afectado la composición de las comunidades de perifiton del río Guadiamar, que se ha traducido en la desaparición de especies que potencialmente se podrían desarrollar en el tramo afectado. Puesto que se ha experimentado con una mezcla de metales aún no se puede establecer cuál es el papel de cada uno de ellos por separado en la estructuración del perifiton. Sin embargo, de forma conjunta, que es como se presentan en el río, producen un impacto al hacer desaparecer a las especies más sensibles. Además, se ha detectado que hay bioacumulación de metales en biofilms. No obstante, el pH es un factor aún más importante en la composición del perifiton en el río.

A pesar del tiempo transcurrido, las comunidades no se han recuperado por completo, siendo aún menor el número de especies en los tramos afectados por el vertido que en las estaciones de control.

\section{AGRADECIMIENTOS}

Este trabajo ha sido financiado por convenio entre la Consejería de Medio Ambiente de la Junta de Andalucía y el CSIC.

\section{BIBLIOGRAFÍA}

ADMIRAAL, W., H. BLANCK, M. BUCKERT-DE JONG, H. GUASCH, N. IVORRA, V. LEHMAN, B.A.H. NYSTRÖM, M. PAULSSON \& R. SABATER. 1999. Short-term toxicity of zinc to microbenthic algae and bacteria in a metal polluted stream. Water Research, 33(9): 1989-1996.

ARAMBARRI, P., F. CABRERA y C. TOCA. 1984. Estudio de la contaminación del río Guadiamar y su zona de influencia (Marismas del Guadalquivir y Coto de Doñana) por residuos de industrias mineras y agricolas. Ed. CSIC. Madrid. $174 \mathrm{pp}$.

CASCO, M. A. (1989). El perifiton del embalse de La Minilla. Relaciones con el fitoplancton y contribución a la producción total. Tesis Doctoral. Universidad de Sevilla. 373 pp.

CASCO, M. A. y J. TOJA. (2003). Efecto de la fluctuación del nivel del agua en la biomasa, la diversidad y las estrategias del perifiton de los embalses. Limnetica, 22(1-2): 115-134.

EICHENBERGER, E., F. SCHLATTER, H. WEILENMANN \& K. WUHRMANN. 1981. Toxic and eutrophicating effects of $\mathrm{Co}, \mathrm{Cu}$ and $\mathrm{Zn}$ on algal benthic communities in rivers. Verh. Internat. Verein. Limnol., 21: 1131-1134.

FOLCH, R. 1985. Història Natural dels Països Catalans, vol. 4. Enciclopedia Catalana S.A. Barcelona. $558 \mathrm{pp}$.

GALLART, F., G. BENITO, J. P. MARTIN-VIDE, A. BENITO, J. M. PRIÓ y D. REGUÉS. 1999. Fluvial geomorfology and hydrology in the dispersal and fate of pirite mud particles released by the Aznalcóllar mine tailing spill. The Science of Total Environment, 242:13-26.

GENTER, R. B. 1996. Ecotoxicology of inorganic chemical stress to algae. Response of algal communities to inorganic stressor. In: Algal Ecology. Freshwater Benthic Ecosystems. Stevenson, R. J.; Bothwell, M. L. \& Lowe, R. L. (eds.): 452-457. Academic Press, San Diego, CA.

GRIMALT, J. O., M. FERRER \& E. MACPHERSON. 1999. The mine tailing accident in Aznalcóllar. The Science of Total Environment, 242: 3-11.

INSERENTANT, R. 1987. quelques réflexions sur la notion de périphyton. Can. Biol. Mar., 28: 297-302

KAPFER, M. 1998 Assessment of the Colonization and Primary Production of Microphytobenthos in the Littoral of Acidic Mining Lakes in Lusatia (Germany). Water, air and soil pollution, 108: $331-340$ 
LELAND, H. V. \& J. L. CARTER. 1984. Effects of copper on species composition of periphyton in a Sierra Nevada, California, stream. Freshwat. Biol., 14:281-296

LELAND, H. V. \& J. L. CARTER. 1985. Effects of copper on production of periphyton, nitrogen fixation and processing of leaf litter in a Sierra Nevada, California, stream. Freshwat. Biol., 15: 155-173.

MOSS, B. 1973. The influence of environmental factors on the distribution of freshwater algae: an experimental study. II. The role of $\mathrm{pH}$ and the carbon dioxide-bicarbonate system. J. Ecol., 61: 157177.

MULHOLLAND, P. J., J. W. ELWOOD, A. V. PALUMBO \& R. J. STEVENSON. 1986. Effects of stream acidification on periphyton composition, chlorophyll and productivity. Can. J. Fish. Aquat. Sci, 43: 1846-1858.

MARKER, A. F. H. \& L. G. WILLOUGHBY. 1988. Epilithic and epiphytic algae in streams of contrasting $\mathrm{pH}$ and hardness. In: Algae and the Aquatic Environment. F. E. Round, (ed.): 312-325. Biopress Ltd., Bristol, UK.

NAVARRO, E., H. GUASCH, I. MUÑOZ, M. REAL $\&$ S. SABATER. 2000. Aplicación de un sistema de canales artificiales en el estudio ecotoxicológico de comunidades microbentónicas. Limnetica, 18:1-14.

NAVARRO, E. 2001. Respuesta ecotoxicológica de comunidades microbentónicas de ríos mediterráneos. Tesis Doctoral. Univ. Barcelona. 116 pp.

NIYOGI, K. D., W. M. LEWIS \& D. M. McKNIGHT. 2002. Effects of stress from mine drainage on diversity, biomass and function of primary producers in mountain streams. Ecosystems, 5: 564-567.

PFEIFER, R. F. \& M. C. DIFFETT. 1975. Some factors affecting primary productivity of stream riffle communities. Archiv für Hydrobiol., 75: 306-317.

PLANAS, D. 1996. Acidification Effects. In: Algal Ecology. Freshwater Benthic Ecosystems. R. J. Stevenson, M. L. Bothwell, \& R. L. Lowe (eds.): 497-522. Academic Press, San Diego, CA.

PRAT, N., J. TOJA, C. SOLÀ, M.D. BURGOS, M. PLANS \& M. RIERADEVAL. 1999. Effect of dumping and cleaning activities on the aquatic ecosystems of the Guadiamar River following a toxic flood. The Sciente of Total Environment, 242: 231-148.

PRAT, N., C. SOLÀ, M. PLANS, J. TOJA y M.D. BURGOS. 2001. La restauración del estado ecológico del río Guadiamar. Medioambiente, 36: 50-55.
PRATT, J. R. \& J. L. ROSENBERGER. 1993. Community change and ecosystem functional complexity: a microcosm study of copper toxicity. In: Environmental Toxicology and Risk Assessment. Vol. 2. F. J. D. J. W. Gorsuch, C.G. Ingersoll \& T. W. La Point. STP (eds.). ASTM. Philadelphia.

PRESCOTT, G. W. 1962. Algae of the Western Great Lakes Areas. W.C. Brown, Co. Dubuque, IA.

SOLÀ, C., M. PLANS, J. TOJA, M. D. BURGOS y N. PRAT. 2001. El accidente de las minas de Aznalcóllar: Efectos sobre el ecosistema acuático del Río Guadiamar. En: De las catástrofes ambientales a la cotidianidad urbana: La gestión de la seguridad y el riesgo. II Coloquio Hispano-canadiense de Barcelona. Geocrítica. Textos de apoyo. Publ. Universidad de Barcelona: 135 pp.

STEVENSON, R. J. \& R. GLOVER. 1993. Effects of algal density and current on ion transport through periphyton communities. Limnol. Oceanogr., 38, 6: 1276-1281.

TOJA, J. \& M. A. CASCO. 1991. Contribution of phytoplankton and periphyton to the production an a reservoir of S.W. Spain. In: Homage to Ramón Margalef; or Why is such pleasure on studying nature. J.D. Ros \& N. Prat (eds.). Oecologia aquatica, 10: 61-76

TOJA, J., E. ALCALÁ, G. MARTÍN, C. SOLÀ, M. PLANS, M. D. BURGOS, A. PLAZUELO y N. PRAT. 2003. Evolución del efecto del vertido tóxico sobre la calidad del agua y de los sedimentos de los ríos Agrio y Guadiamar. En: Ciencia y restauración del río Guadiamar. J. M. Arenas, F. R. Matínez, A. Mora, C. Montes \& F. Borja (eds.). Consejería de Medio Ambiente. Junta de Andalucía: 78-93.

UTERMÖHL, H. 1958. Zur Vervollkommnung der quantitativen Phytoplankton-Methodik. Mitt. Int. Verein. Limnol., 9: 1-38.

VINEBROOKE R. D. \& M. D. GRAHAM. 1997. Periphyton Assemblages as Indicators of Recovery in Acidified Canadian Shield Lakes. Can. J. Fish. Aquat. Sci., 54: 1557-1568.

VOLLENWIDER, R. A. 1969. Primary production in aquatic environments. IBP. Handbook 12: 213 pp. Blachwell Sci. Oxford.

WETZEL, R. G. 1983. Opening remarks. En: Periphyon of freshwater ecosystems. Wetzel R. G. (ed.). 3-4. Dr. Junk Publishers. The Hague.

WHITFORD, L. A. \& G. J. SCHUMACHER. 1961. Effects of current on mineral uptake and respiration by a fresh-water alga. Limnol. Oceanogr., 6: 423425. 
